\title{
Strontium-incorporated titanium implants promote bone-implant osseointegration in osteoporotic rabbits
}

Guo-fen Lin, Chuan Zhou, An-tian Xu, Fu-ming He

Affiliated Stomatology Hospital, School of Medicine, Zhejiang University, Hangzhou 310006, China

\section{Background and Aim}

Implant failure may occur in patients with osteoporosis, especially in elderly over 50 years including postmenopausal females ${ }^{1}$. This osteoporosis leads to insufficient bone-implant contact and can severely deteriorate the primary stability and osseointegration establishment of dental implant. Recently, the positive effects of Strontium (Sr) iron on inhibiting bone resorption and promoting bone formation have been elucidated in numerous studies. Our previous in vitro and vivo studies incorporated $\mathrm{Sr}$ iron onto the surface of SLA implant by hydrothermal treatment, and the results demonstrated that Sr-incorporated surfaces has a positive influence on mesenchymal stem cell (MSC) differentiation and enhancing implant osseointegration in healthy animals. The aim of this study was designed to compare the osseointegration behavior of conventional SLA implants and Sr-SLA implants in ovariectomized rabbits ${ }^{2}$.

\section{Methods and Materials}

Thirty-two New Zealand rabbits, half of which were carried out with bilateral ovariectomy and glucocorticoid administration for six weeks (OVX group), and another 16 rabbits were conducted with SHAM operation as for control group. Bone mineral density of lumbar spines (L3-L5), global knee bone and subchondral bone was measured before and after ovariectomy or SHAM operations using dual energy Xray absorptiometry(Fig.1). After successful osteoporotic-induced model, the SLA and Sr-SLA implants were randomly inserted into the tibia and femur metaphysis of each animal. The rabbits were respectively sacrificed 3 and 6 weeks after dental implant placement. The samples from femur metaphysis in each group at each time point were subjected to removal torque testing, and the remaining samples from tibia metaphysis were conducted for histomorphometric analysis ${ }^{3}$.
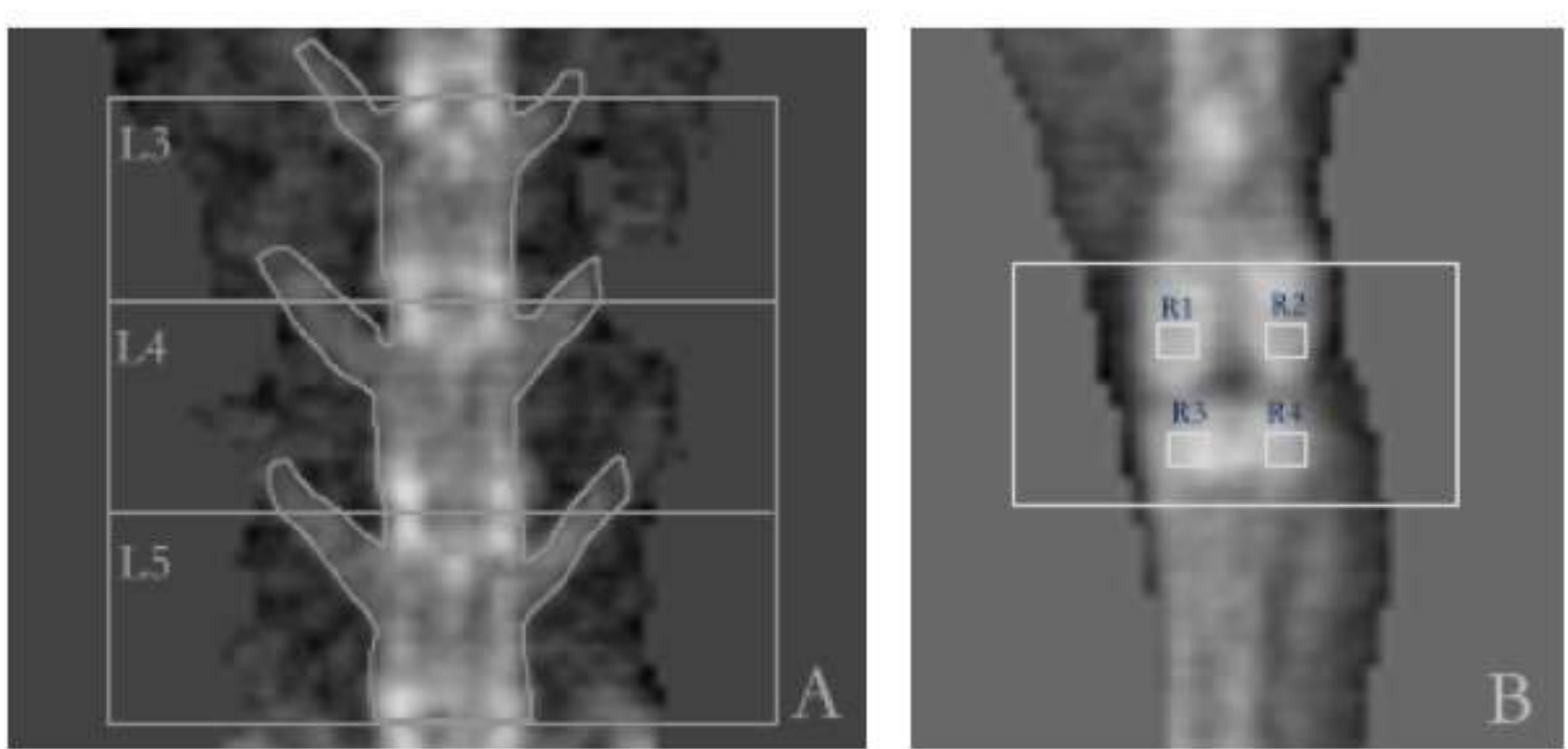

Fig.1 A: The mean of the third to fifth vertebrae absorptiometric values were calculated for lumbar spines. B: The global knee was selected to evaluate trabecular and cortical bone (large rectangle). Four squared areas (R1-R4) adjacent to the joint line were used to assess subchondral bone in each femoral condyle and tibial plateaux.
At the end of the healing period, all implants were osseointegrated and histologically in direct contact with the surrounding bone. The Sr-SLA implants displayed closer contact and much more newly formed bone than the SLA implants in OVX rabbits(Fig.2). At 3 weeks (Fig.3), the removal torque values (RTV) of the Sr-SLA implants were significantly higher than those of the SLA implants in both OVX $(P<0.05)$ and healthy animals $(P<0.05)$. At 6 weeks, the RTV of the Sr-SLA implants were also significantly larger than those of the SLA implants in both OVX $(P<0.05)$ and healthy animals $(P<0.05)$. At 3 weeks, the Sr-SLA implants presented significantly higher bone-to-implant contact (BIC) than the SLA implants in osteoporotic animals in both cortical bone $(80 \pm 6.6$ vs. $70.2 \pm 4.4, P<0.05)$ and cancellous bone $(31.3 \pm 7.6$ vs. $20.1 \pm 6.0, P<0.01)$. The bone area (BA) of the Sr-SLA implants $(37.2 \pm 10.7)$ showed significantly higher than the SLA implants $(26.2 \pm 7.2)$ in osteoporotic animals in the cortical bone $(P<0.05)$, whereas there was no statistical difference between the Sr-SLA implants $(10.2 \pm 2.3)$ and SLA implants (8.2 1.7$)$ in the cancellous bone. At 6 weeks, the BIC and BA analysis exhibited no statistical significant difference between the four groups.
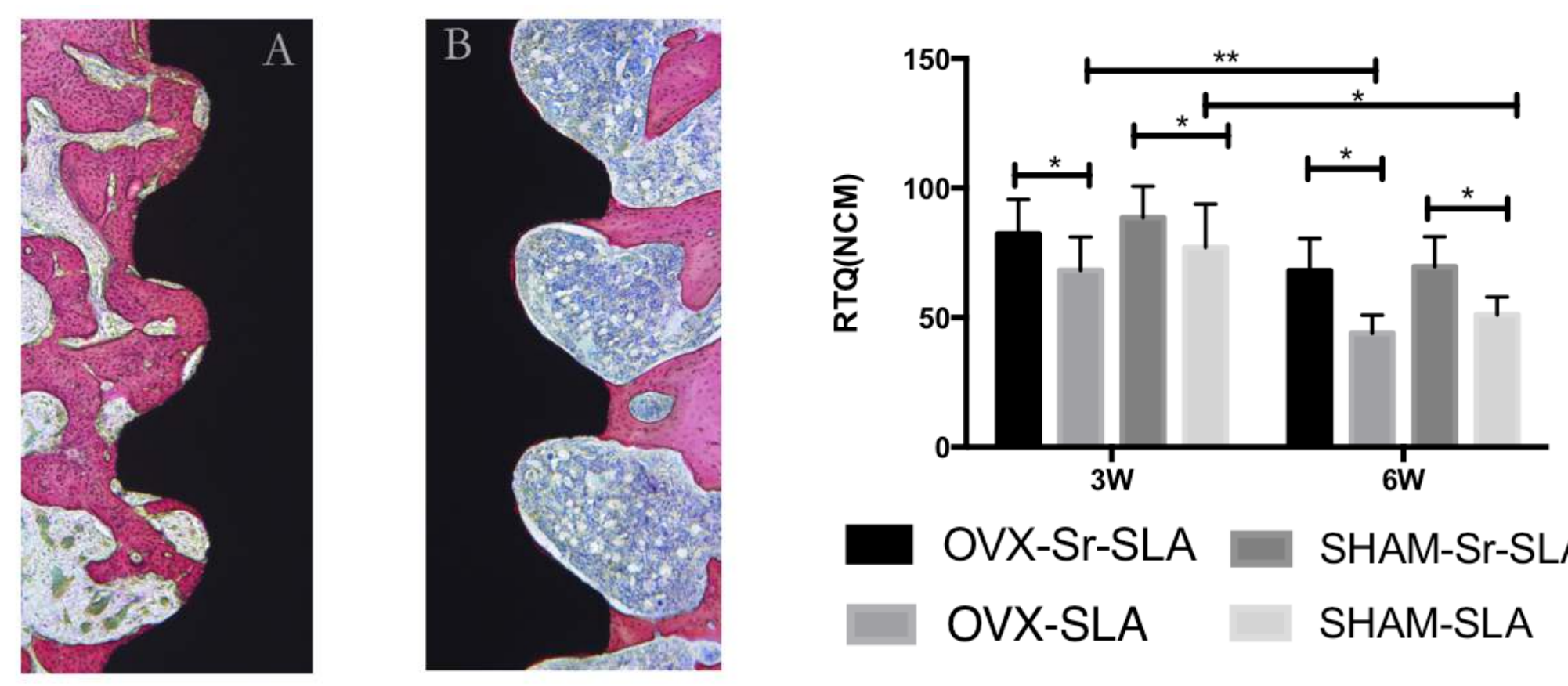

OVX-Sr-SLA SHAM-Sr-SLA

OVX-SLA SHAM-SLA

Fig.2 The Sr-SLA implants(A) Fig.3. The mean and standard displayed closer contact and deviation of RTV $(\mathrm{Ncm})$ for the much more newly formed four groups at 3 and 6 week. * $P$ bone than the SLA implants(B) $<0.05$, ${ }^{* *} \mathrm{P}<0.01$ in OVX rabbits. Original magnification $\times 40$.

\section{Conclusions}

Considering the results obtained from the present study, it is suggested that Sr-incorporated implant has a better effect on bone osseointegration than conventional SLA implants in both osteoporotic and non-osteoporotic animals. In addition, Sr-incorporated implant notably plays an important role in the early period of bone osseointegration.

\section{References}

1. Bryant SR, Zarb GA. Outcomes of implant prosthodontic treatment in older adults. J Can Dent Assoc 2002; 68: 97-102.

2. Fu DL, Jiang QH, He FM, Yang GL, Liu L. Fluorescence microscopic analysis of bone osseointegration of strontiumsubstituted hydroxyapatite implants. J Zhejiang Univ Sci B 2012; 13: 364-371

3. Martin-Monge E, Tresguerres IF, Blanco L, Khraisat A, Rodriguez-Torres $\mathrm{R}$, Tresguerres JA. Validation of an osteoporotic animal model for dental implant analyses: an in vivo densitometric study in rabbits. Int $\mathrm{J}$ Oral Maxillofac Implants 2011; 26: 725-730. 